\title{
Kindliche Unterarmfrakturen
}

N eben dem Spiel- und Raufverhalten hat die große Popularität von Modesportarten wie Inlineskating, Skate- und Snowborden in den letzten Jahren bei Kindern und Jugendlichen zu einer erheblichen Steigerung von Verletzungen auch im Bereich der oberen Gliedmaßen geführt. Besonders betroffen waren dabei die kindlichen Unterarme. Dies bedeutet, daß sich der Behandler mit den aktuellen Fragen und Problemen der Versorgung kindlicher Unterarmfrakturen befassen muß.

Bei Durchsicht der älteren Lehrbücher ist auffällig, daß einheitlich die Meinung vertreten wird, daß über $90 \%$ aller kindlichen Unterarmfrakturen mit gutem Ergebnis unter konservativer Behandlung zur Ausheilung gebracht werden können. Inzwischen sind durch Neuentwicklungen aber auch den Zeitgeist zahlreiche Wandlungen eingetreten.

Trotz der oft guten Reparationsfähigkeit des wachsenden Skeletts mit altersentsprechend beachtlichen Spontankorrekturen werden heute von Eltern und "Nachbehandlern" Achsabweichungen, die sich erst im Laufe von Jahren zurückbilden, immer weniger akzeptiert. In vielen Fällen wird auch deshalb einer operativen Versorgung der Vorzug gegeben.
Auf der anderen Seite sind optimale Behandlung und optimales Ergebnis nicht mit operativer Therapie gleichzusetzen. Bei Behandlungsbeginn muß geklärt werden, welches therapeutisches Vorgehen für die vorliegende Fraktur geeignet ist. Das Leitthema dieses Heftes beschäftigt sich daher mit der differenzierten Therapie der kindlichen Unterarmfrakturen, stellt die Indikationen zur operativen Therapie dar, zeigt aber auch, in welchen Fällen die bewährte konservative Therapie nicht aufgegeben werden sollte.

Immer muß dabei die Behandlung des Kindes insgesamt im Zentrum stehen, der durch den Unfall verursachte Schaden darf durch iatrogene Bemühungen nicht verschlimmert werden. Minimalinvasive Eingriffe haben bei gegebener Indikation zur Operation Priorität und tragen dazu bei, stationäre Behandlungen möglichst kurz zu halten. Daß die Qualität der Behandlung mit diesem Vorgehen gesteigert werden kann, beweisen die Publikationen der letzten Jahre.

\section{P. Hahn}

